\title{
What physicians and patients should know about different designs of cochlear implant electrodes?
}

\author{
Mohamed S Elgandy ${ }^{1,2 *}$ and Mohammad K Mobashir ${ }^{1}$ \\ ${ }^{1}$ Department of Otolaryngology-Head and Neck surgery, Zagazig University, Egypt \\ ${ }^{2}$ Department of Otolaryngology-Head and Neck Surgery, University of Iowa, Iowa City, USA
}

\begin{abstract}
Cochlear implants have changed how we consider hearing loss. Over the years, selection criteria have moved from profound to severe to moderate hearing loss. Now those with good residual low-frequency hearing, or only a unilateral hearing loss can benefit. Several exciting advances have been made in the last few years. A combination of technological advancements in device hardware, processing capabilities, improved surgical techniques and electrode factors, such as correct scala placement and modiolar proximity were found to correlate positively with patient outcomes. This is an important consideration as the electrode forms a permanent and lifelong interface between the cochlear implant delivering stimulation and the neural structures of the cochlea. This paper reviews different electrodes types and future considerations in electrode design in order to improve overall patient quality of patient's life.
\end{abstract}

\section{Introduction}

Cochlear implant (CI) has successfully restored hearing, improving the quality of life in adults and providing candidates with normal developmental and educational opportunities. Although improvements in coding strategies [1-4] and advancements in speech processors [5] have resulted in an overall improvement in the performance of the $\mathrm{CI}$, the electrode considered as an important element as it comes into direct contact with the intra-cochlear tissues that line the neural elements [6].

For a CI user to fully benefit from their device, the following factors need to be considered: (i) electrode array insertion depth and cochlear coverage [7], (ii) matching neuro-tonotopicity, (iii) atraumatic electrode array insertion and insertion forces against the intra-cochlear structures [8] and (iv) choosing an electrode array that matches the recipient's individual cochlear anatomy especially when the anatomy is malformed and a regular CI electrode will not give an optimal placement closer to the neural structures.

It is important to remember that a damaged electrode can be replaced, but a damaged cochlea cannot be replaced at any cost. Keeping this in mind, makes it so difficult to achieve an "ideal" electrode design. All the major CI manufacturers have their own electrode design within their manufacturing know how which they feel is the best for bringing better hearing outcomes to the patients.

\section{Lateral wall electrodes}

In Early CI studies, the primary goal of implantation was to obtain effective stimulation of the auditory system.

Early multichannel CI electrodes were all straight in design ("lateral wall" electrodes), containing between 8 and 22 electrode contacts and up to $1.3 \mathrm{~mm}$ in diameter. The size and stiffness of these early lateral wall electrodes were a function of the technology at the time, and they supported the surgical aims to place a multichannel electrode within the cochlea to deliver electrical stimulus to the auditory nerve.
These stiffer lateral wall electrodes could result in the application of more force onto the surrounding blood vessels and, therefore, limit the blood supply to the neural elements that extend into the apical portion of the cochlea where residual hearing is typically located [9]. An electrode array which is bigger in dimension would take up more volume in the perilymph and could also produce a higher intracochlear pressure depending on the speed of electrode insertion into the cochlea [10]. However, a bigger electrode array would also bring the stimulating contacts closer to the neural elements, so a balance should be determined how far the stimulating contacts should be positioned from neural tissue.

With the introduction concept of both soft surgery and combined electrical and acoustic hearing, manufacture's goal was to preserve the cochlea structure and potentially preserve residual low-frequency hearing in patients who had some levels of functional hearing but were not benefiting from auditory amplification via hearing aids alone. This resulted in the development of thinner, shorter, and more flexible lateral wall electrodes, such as the Hybrid-L24 and Slim Straight electrodes (Cochlear Ltd., Sydney Australia), the Flex series (MEDEL, Innsbruck Austria) and Slim (Advanced Bionics, Valencia USA). These lateral wall electrodes were developed with smaller diameters to facilitate round window insertion and shorter lengths to minimize trauma and total loss of residual low-frequency hearing [11,12].

Lateral wall electrode lengths had developed over time. There is convergence to a lateral wall length of $20 \mathrm{~mm}$ (approximately $360^{\circ}$ )

${ }^{\star}$ Correspondence to: Mohamed Salah Elgandy, Department of OtolaryngologyHead and Neck Surgery, University of Iowa, Iowa City, USA, Tel: +1 (319)5193862; E-mail: melgandy@healthcare.uiowa.edu

Key words: cochlear implant, electrode, straight and precurved, future technology

Received: September 09, 2019; Accepted: September 23, 2019; Published: September 27, 2019 
to minimize trauma and preserve functional hearing and $25 \mathrm{~mm}$ (approximately $450^{\circ}$ ) to preserve structure and maximize coverage of the spiral ganglion population for electrical stimulation. Figure 1 also highlights two very different approaches taken that have ultimately arrived at the current approach of a $20-25 \mathrm{~mm}$ insertion depth, balancing both cochlear trauma with cochlear coverage [13].

The height of the lateral aspect of scala tympani is an important metric in defining the maximum apical diameter of a lateral wall electrode, which is required to fit within this space without interference or placing upward pressure on the basilar membrane. This scala height, relative to electrode diameter becomes more critical apically as the basilar membrane is not a homogeneous structure with consistent properties along its length, but progressively increases in width while decreasing in thickness and stiffness from base of the cochlea to the apex $[14,15]$.

The maximum electrode diameter therefore that fits within the lateral aspect where the basilar membrane joins the spiral ligament at $450^{\circ}$ is approximately $0.4 \mathrm{~mm}$, reducing to approximately $0.3 \mathrm{~mm}$ at $540^{\circ}[16,17]$. The smallest apical dimension (vertical height) of current commercially available lateral wall electrodes is $0.35 \mathrm{~mm}$ (Hybrid L24 and Slim Straight electrodes from Cochlear Ltd.). Figure 2 shows the apical heights of all current commercially available lateral wall electrodes.

Lateral wall electrodes have a typical force profile during electrode insertion, that is, initial impact and increase in force as the electrode contacts the lateral wall (between $150^{\circ}$ and $180^{\circ}$ ), followed by an exponential increase as the electrode travels around the narrowing spiral of the cochlea and friction between the soft tissue and silicone of the electrode increases. As there is no recognized standard providing guidance on insertion force testing, there have been a wide range of reported lateral wall electrodes insertion forces. Ideally, an electrode

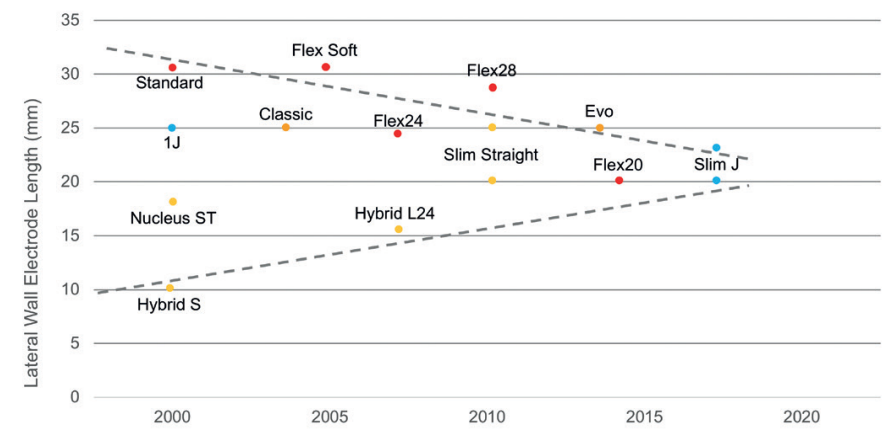

Figure 1. Trend in lateral wall electrode lengths over time

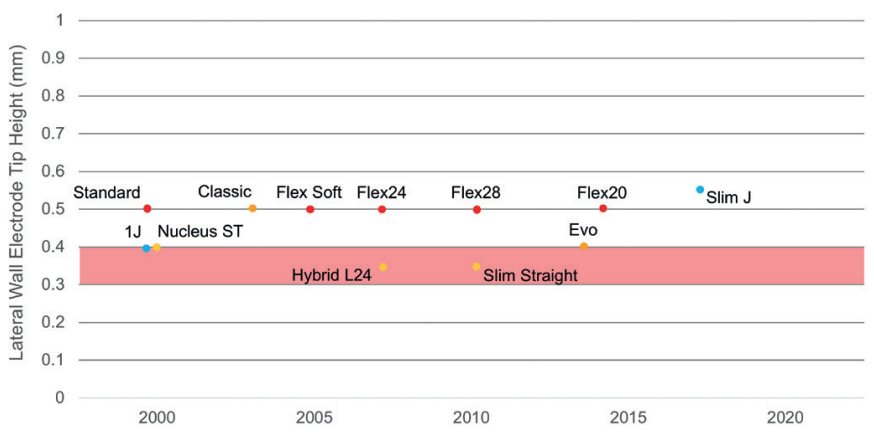

Figure 2. Apical heights of all current commercially available lateral wall electrodes array should be designed to allow for insertion without damaging any of the intra-cochlear structures. However, typically a certain degree of trauma is to be expected with all the currently available electrode designs. Trauma to structures of the lateral wall is one of the most commonly reported types of intraoperative insertional damage by straight electrodes.

Buckling or kinking of the electrode in the basal turn, resulting in basal fracture of the osseous spiral lamina, is another commonly reported mechanism of trauma with flexible lateral wall electrodes. This was first reported in 1997 [18], The authors postulated that basal buckling occurs when the insertion beyond resistance is attempted, causing electrode buckling and trauma to the osseous spiral lamina or basilar membrane in the lower basal turn. Insertion beyond resistance, typically the result of the lateral wall electrode tip becoming impinged against the spiral ligament, or friction against the underside of the basilar membrane, resulted in the basal electrode buckling and corresponding fracture to the osseous spiral lamina in the basal turn.

Post-operative changes to the intracochlear environment are also important to consider, especially if the aim is functional hearing preservation. If atraumatic electrode insertion with a lateral wall electrode is achieved, the electrode will always be positioned directly adjacent and in a very close proximity to the basilar membrane. Intracochlear fibrosis consisting of inflammation, fibrosis, and neo osteogenesis will form around the electrode array due to the opening of the cochlea, foreign body tissue response, or disruption of any soft tissue or venous structure of scala tympani during insertion [19]. Changes to the intracochlear environment have been reported in several histopathological studies of temporal bone specimens donated by deceased CI patients [20-22]. The build-up of fibrosis around the electrode over time will potentially impact or form a connection to the spiral ligament and basilar membrane, which will result in mechanical impedance with reduction or complete loss of hearing over time.

Post-operative changes are also important when considering the explanation of electrode arrays, especially considering that in young children, explanation may be expected during their lifetime. An in vitro study [23] utilized a plastic cochlea model backfilled with a gel formulation representing soft tissue surrounding the electrode array. It was found that explant trauma, measured as disruption to the gel surrounding the electrode correlated with the depth of insertion, with deeper insertion of a lateral wall electrode, resulting in greater disruption. When $28 \mathrm{~mm}$ electrode is explanted, the load forces the electrode medially so that the electrode contacts the modiolus from approximately $300^{\circ}$ with significant disruption of the surrounding gel as the electrode moves from a lateral to a medial position. In contrast, a shallower $20 \mathrm{~mm}$ insertion remains lateral within the electrode lumen due to reduced load and friction, with only minor disruption of the surrounding gel.

\section{Perimodiolar electrode}

Pre-curved electrodes are designed to be positioned close to the modiolar wall. Due to the pre-curved design, the electrode hooks around the modiolus wall and prevents it from extruding when micromovements occur in the electrode lead [24]. It is also easier to insert than using a straight lateral wall electrode.

Early studies on perimodioar electrodes found that placing these electrodes close to the medial margin of scala tympani, demonstrated reduced Electrical Auditory Brainstem Response (EABR) thresholds in a cat model relative to electrodes positioned along the lateral wall, 
with the EABR threshold reducing as the electrode array was moved from a lateral to a medial position [25] helped all three commercial CI companies (Cochlear Ltd., Advanced Bionics and MED-EL) to actively develop perimodiolar electrode arrays, with each taking very different approaches to achieve modiolar proximity: Cochlear Ltd. with the Contour electrode (stylet based), Advanced Bionics with the Hi-Focus electrode (positioner based), and MED-EL with the Peri-Modiolar Electrode (push-wire based) designs. Although being different in design, all three achieved a final position close to the modiolus. All were initially inserted straight, achieving full insertion as a lateral wall electrode, and only after the initial insertion did the electrodes achieve a final position closer to the modiolus [26,27].

\section{Stylet-based perimodiolar arrays}

It was found that type of electrodes is more likely to cause trauma than any type of electrodes due to the presence of a stylet wire which brings the pre-curved electrode to be in a straight configuration before complete insertion occurs [28]. During insertion, because the electrode tip is stiff with the stylet in place, the electrode could penetrate the spiral ligament at the end of the straight portion of the basal turn of the cochlea. Recently developed perimodiolar electrodes on the other hand were larger in diameter, and so they typically required a separate cochleostomy. The Contour Advance (Cochlear Ltd.) was developed soon after as a variant of the original Contour electrode, but with a unique Advance off Stylet (AOS) surgical technique, where the electrode is partially inserted into the basal turn only, and the electrode then is advanced off the stylet. This insertion technique was specifically developed to reduce contact with, and therefore trauma to, the structures of the lateral wall during initial insertion [29]. With the AOS insertion technique, the electrode avoids contact with the lateral wall completely and therefore the insertion forces remain near zero throughout the insertion. on the other hand the Standard Insertion Technique (SIT), where the electrode is inserted fully in the same way as a lateral wall electrode prior to the stylet being removed having a similar insertion force profile to lateral wall electrodes, that is, as the electrode contacts the lateral wall, the insertion forces increase significantly, risk of trauma here is higher due to the relative stiffness of the electrode with the stylet in place.

The apical electrode diameter of current stylet-based perimodiolar electrodes is $0.5 \mathrm{~mm}$ (Contour Advance from Cochlear Ltd.; Mid scala from Advanced Bionics), which is a technical constraint created by the requirement of an internal stylet. Although a $0.5 \mathrm{~mm}$ apical electrode dimension is not dissimilar to the dimensions of contemporary lateral wall electrodes. space within the narrowing scala tympani is not the concern, but rather the ability to insert via round window. So, a separate cochleostomy has typically been required, which then exposes the risk of incorrect cochleostomy placement [30] and in many cases an anterior cochleostomy directly into scala vestibule or contributing to early translocation from scala tympani to vestibuli [31].

\section{Sheath-based perimodiolar electrode}

More recently, the Slim Modiolar Electrode (CI532 implant) from Cochlear Ltd. introduced a new concept of a "sheath-based" perimodiolar electrode, as opposed to "stylet-based". This difference in approach to straighten and insert a pre-curved electrode is a significant advancement that addresses the two main challenges identified as contributing to the higher rates of trauma with stylet-based perimodiolar electrodes, being 1) variability or compliance with the AOS insertion technique, and 2) the ability to insert via the round window. The sheathbased design also allows for ease of reloading the electrode if required.
The sheath design removes the need for an internal stylet allowing for a significantly thinner electrode array, with apical dimension of 0.35 $\mathrm{x} 0.4 \mathrm{~mm}$ and $0.5 \mathrm{~mm}$ basally, which is smaller than or equivalent to contemporary lateral wall electrodes. The soft and flexible sheath design therefore allows for round window insertion, ensuring placement in the scala tympani.

Advances in the design of perimodiolar electrodes, specifically moving from a stylet-based design to a sheath-based design for the Slim Modiolar electrode (CI532), has resulted in a much thinner and more flexible pre-curved electrode array. This approach provides a perimodiolar electrode that is 1) suitable for round window insertion, 2) ensures $100 \%$ compliance with a surgical technique that avoids electrode contact with the lateral wall and basilar membrane, while 3) positioning the electrodes in close proximity to the auditory nerve for optimal electrical stimulation. The SME therefore provides significant advantages over stylet-based designs, with the potential to preserve structure as reported in early clinical studies [32-34].

\section{Emerging electrode technologies}

- Drug-eluting electrode arrays show a lot of potential due to being atraumatic whilst enabling the drug components to act on the intracochlear neural elements. As the lifetime of any $\mathrm{CI}$ is typically less than twenty years, many CI recipients will require explantation and re- implantation during their lifetime. Therefore, it is important to provide an atraumatic solution to protect the intracochlear anatomical structures and to prevent further fibrotic tissue growth within the cochlea [35].

- Nanoparticles coated electrodes: the miniaturization of drug carriers down to nanoscale level has led to strategies being devised whose aim is by using nanoparticles to allow CI based release of drugs for local therapy of the inner ear. Nanoparticles, functioning as non-viral vectors of biogenic agents (e.g. genes, neurotrophic factors and steroid sequences), protected from the effects of the body's metabolism, are to be transported specifically to the desired target location and time released. Integrating a minute (nanoscale) drug depot into a CI could for example, under this approach, lead to targeted release of neurotrophic factors and eventually to an improvement in nerve-electrode interaction. The uptake of nanoparticles has already been demonstrated in the inner ear [36], as has the fundamental biocompatibility of the particles used in terms of preserving inner-ear structures and their function [37].

- Optical CI arrays: as electrodes work by stimulating spiral ganglion nerves by electrical stimulation. The implant arrays contain multiple electrode channels to assist with better defined sounds; however, it is difficult to isolate the signal to specific neural groups. At the time of surgery, the implant array is blindly inserted within the cochlea, leading to potential suboptimal positioning of the electrodes over ganglion cells within the intended frequency range. This suboptimal positioning leads to interference across electrode channels, contributing to the struggle with speech reception, especially in noisy environments, among CI recipients [38]. Kallweit et al. [39] showed the ability to optically stimulate the cochlea using lasers in guinea pigs. Also, optical electrodes using infrared light to selectively stimulate cochlear neurons in cats have been described [40]. In addition, optogenetics is a technology that has been investigated in animal models and uses light to activate neuronal light-sensitive ion channels in genetically engineered neurons [40]. A potential outlook for CI involves arrays composed of multiple light-emitting segments, which could improve on the broad activation associated 
with the current electrical stimulation pathways, continued research within these technological advancements and demonstration of safety may lead to human clinical applications in the future.

- Human biohybrid electrodes: Roemer et al. [41] reported a study in which biohybrid electrodes utilizing autologous mononuclear cells (MNC) isolated from bone marrow was developed and tested in three patients. They can be derived from peripheral blood or from the bone marrow and consist of different cell types including hematopoietic and mesenchymal progenitor cells. The unique capacity of progenitor cells to exert different therapeutic actions depending on the context in which they are transplanted has been reviewed recently relying on the ability of human BM-MNC to release cytokines, chemokines, and growth factors that exert anti-inflammatory and neuroprotective effects [42,43]. Intrinsic properties of progenitor cells allow for the modulation of the immune system and enable complete regeneration without scar formation, neuroprotection and protection of residual hearing.

In that study, using fibrin adhesive as a carrier for BM-MNC, a simple and effective cell coating procedure for CI electrodes was developed that can be utilized on-site in the operating room for the generation of biohybrid electrodes for intracochlear cell-based drug delivery. The short processing time for isolation of BM-MNC from the bone marrow did not induce any delay in surgery. The coating procedure was simple and was performed by the surgeon immediately prior to insertion. Insertion was uncomplicated. Initial safety requirements have been met in all cases presented here after 6 months, demonstrating a lack of complications and the clinical feasibility of the approach. Further investigations will concentrate on the enhancement of the effect of BM-MNC.

- Robotic electrode insertion: In addition to contribution in drilling, robots have been shown to assist with electrode insertion, studies have shown that average robotic insertion forces and manual insertion forces by surgeons are similar $\left(0.005 \_0.014 \mathrm{~N}\right.$ for robots and $0.004 \_0.001 \mathrm{~N}$ for surgeons using the Advance Off-Stylet technique), but peak insertion forces between 120 and 200 are much higher with surgeons. Decreasing those peak forces would be significant in minimizing trauma induced damage [44]. Robots designed to sense insertion forces and modify their trajectory [45]. Zhang et al. [46] took it one step further with a robot that can steer the electrode through the scala tympani to decrease the maximum insertion force by $59.6 \%$.

\section{Conclusions}

The ideal array would have the following qualities: (i) it could be inserted through either the cochleostomy or the RW opening, (ii) it would be available in a range of different lengths to accommodate each individual cochlea in terms of insertion depth and in covering the middle turn of the cochlea in order to cover the complete range of SG cells, (iii) it could be inserted without complications using the stylet/ loading/insertion tool, and (iv) the array would not cause any major trauma to any of the intra-cochlear structures. Until this "ideal" array is developed, the available data shows that straight, flexible LW electrodes fit the criteria of being an optimum array of choice. New emerging electrode designs provide hope not only for hearing preservation but also for reducing inflammatory and fibrotic process over years in implanted candidates.

\section{References}

1. Lopez-Poveda EA, Eustaquio-Martin, A, Stohl JS, Wolford RD, Schatzer, R, et al. (2016) A Binaural Cochlear Implant Sound Coding Strategy Inspired by the Contralateral Medial Olivocochlear Reflex. Ear Hear 37: 138-148. [Crossref]

2. Nogueira W, Rode T, Buechner A (2016) Spectral contrast enhancement improves speech intelligibility in noise for cochlear implants. J Acoust Soc Am 139: 728-739.

3. Riss D, Hamzavi JS, Blineder M, Honeder C, Ehrenreich I, et al. (2014) FS4, FS4-p and FSP: a 4-month crossover study of 3 fine structure sound-coding strategies. Ear Hear 35: e272-e281. [Crossref]

4. Adams D, Ajimsha KM, Barberá MT, Gazibegovic D, Gisbert J, et al. (2014) Multicentre evaluation of music perception in adult users of Advanced Bionics cochlear implants. Cochlear Implants Int 15: 20-26. [Crossref]

5. Dazert S, Thomas JP, Buechner A, Mueller J, Hempel JM, et al. (2017) Off the ear with no loss in speech understanding: comparing the RONDO and the OPUS 2 cochlear implant audio processors. Eur Arch Otorhinolaryngol 274: 1391-1395. [Crossref]

6. Wanna GB, Noble JH, Carlson ML, Gifford RH, Dietrich MS, et al. (2014) Impact of Electrode Design and Surgical Approach on Scalar Location and Cochlear Implant Outcomes. Laryngoscope 124: S1-S7. [Crossref]

7. O'connell BP, Hunter JB, Haynes DS, Holder JT, Dedmon MM, et al. (2017) Insertion depth impacts speech perception and hearing preservation for lateral wall electrodes. Laryngoscope 127: 2352-2357. [Crossref]

8. Mady LJ, Sukato DC, Fruit J, Palmer J, Catherine R, et al. (2017) Hearing Preservation: Does Electrode Choice Matter? Am Otolaryngol Head Neck Surg 157: 1-11.

9. Mom T, Chazal J, Gabrillargues J, Gilain L, Avan P (2005) Cochlear blood supply: an update on anatomy and function. $\operatorname{ORL} \operatorname{Fr} 88: 82$

10. Mittmann P, Mittmann M, Ernst A, Todt I (2017) Intracochlear Pressure Changes due to 2 Electrode Types: An Artificial Model Experiment. Otolaryngol Head Neck Surg 156: 712-716. [Crossref]

11. Lenarz T, Stöver T, Buechner A, Lesinski-Schiedat A, Patrick J, et al. (2009) Hearing conservation surgery using the Hybrid-L electrode. Results from the first clinical trial at the Medical University of Hannover. Audiol Neurootol 14: S22-S31. [Crossref]

12. Skarzynski H, Lorens A, Matusiak M, Porowski M, Skarzynski PH, et al. (2014) Cochlear implantation with the nucleus slim straight electrode in subjects with residual low-frequency hearing. Ear Hear 35: 33-43. [Crossref]

13. Brant JA, Ruckenstein MJ (2016) Electrode selection for hearing preservation in cochlear implantation: A review of the evidence. World J Otorhinolaryngol Head Neck Surg 2: 157-160. [Crossref]

14. Dallos P (1992) The active cochlea. J Neurosci 12: 4575-4585. [Crossref]

15. Echteler SM (1995) Structural correlates of frequency-place map development. Abstr Assoc Res Otolaryngol 18: 442.

16. Rebscher SJ, Hetherington A, Bonham B, Wardrop P, Whinney D, et al. (2008) Considerations for design of future cochlear implant electrode arrays: electrode array stiffness, size, and depth of insertion. J Rehabil Res Dev 45: 731-747. [Crossref]

17. Avci E, Nauwelaers T, Lenarz T, Hamacher V, Kral A (2014) Variations in microanatomy of the human cochlea. J Comp Neurol 522: 3245-3261. [Crossref]

18. Gstoettner W, Plenk H Jr, Franz P, Hamzavi J, Baumgartner W, et al. (1997) Cochlear implant deep electrode insertion: extent of insertional trauma. Acta Otolaryngol 117: 274-277. [Crossref]

19. Roland PS, Wright CG (2006) Surgical aspects of cochlear implantation: mechanisms of insertional trauma. Adv Otorhinolaryngol 64: 11-30. [Crossref]

20. Nadol JB Jr, Eddington DK (2004) Histologic evaluation of the tissue seal and biologic response around cochlear implant electrodes in the human. Otol Neurotol 25: 257-262. [Crossref]

21. Li PM, Somdas MA, Eddington DK, Nadol JB Jr. (2007) Analysis of intracochlear new bone and fibrous tissue formation in human subjects with cochlear implants. Ann Otol Rhinol Laryngol 116: 731-738. [Crossref]

22. Fayad JN, Makarem AO, Linthicum FH Jr. (2009) Histopathologic assessment of fibro $\neg$ sis and new bone formation in implanted human temporal bones using 3D reconstruction. Otolaryngol Head Neck Surg 141: 247-252. [Crossref]

23. Roland JT Jr, Risi F (2016) A model to evaluate electrode explant force and trauma Presented at CI2016. Toronto, Canada. 
24. Mittmann P, Rademacher G, Mutze S, Ernst A, Todt I (2015) Electrode Migration in Patients with Perimodiolar Cochlear Implant Electrodes. Audiol Neurotol 20: 349-353. [Crossref]

25. Shepherd RK, Hatsushika S, Clark GM (1993) Electrical stimulation of the auditory nerve: the effect of electrode position on neural excitation. Hear Res 66: 108-120. [Crossref]

26. Gstoettner WK, Adunka O, Franz P, Hamzavi J Jr, Plenk H Jr, et al. (2001) Perimodiolar electrodes in cochlear implant surgery. Acta Otolaryngol 121: 216-219. [Crossref]

27. Eshraghi AA, Yang NW, Balkany TJ. (2003) Comparative study of cochlear damage with three perimodiolar electrode designs. Laryngoscope 113: 415- 419. [Crossref]

28. Rebscher S, Hetherington A, Bonham B, Wardrop P, Whinney D, et al. (2008) Considerations for design of future cochlear implant electrode arrays: electrode array stiffness, size, and depth of insertion. J Rehabil Res Dev 45: 731-747. [Crossref]

29. Roland JT Jr. (2005) A model for cochlear implant electrode insertion and force evaluation: results with a new electrode design and insertion technique. Laryngoscope 115: 1325-1339. [Crossref]

30. Adunka OF, Buchmann CA (2007) Scala tympani cochleostomy I: results of a survey. Laryngoscope 117: 2187-2194. [Crossref]

31. Aschendorff A, Kromeier J, Klenzner T, Laszig R (2007) Quality control after insertion of the nucleus contour and contour advance electrode in adults. Ear Hear 28: 75S-79S [Crossref]

32. Cuda D, Murri A (2017) Cochlear implantation with the nucleus slim modiolar electrode (CI532): a preliminary experience. Eur Arch Otorhinolaryngol 274: 41414148. [Crossref]

33. McJunkin JL, Durakovic N, Herzog J, Buchman CA. (2018) Early outcomes with a slim, modiolar cochlear implant electrode array. Otol Neurotol 39: 28-33. [Crossref]

34. Ramos-Macías A, Borkoski-Barreiro SA, Falcón-González JC, Ramos-de Miguel A. (2017) Hearing Preservation with the Slim Modiolar Electrode Nucleus CI532 Cochlear Implant: A Preliminary Experience. Audiol Neurootol 22: 317-325. [Crossref]
35. Audiology Online (2016) New research shows drug-releasing electrodes likely to improve cochlear implant performance. Available from: http://www.audiologyonline. com/releases/new-research-shows-drug-releasing-18081.

36. Zou J, Saulnier P, Perrier T, Zhang Y, Manninen T, et al. (2008) Distribution of lipid nanocapsules in different cochlear cell populations after round window membrane permeation. J Biomed Mater Res B Appl Biomater 87: 10-18. [Crossref]

37. Tamura T, Kita T, Nakagawa T, Endo T, Kim TS, et al. (2005) Drug delivery to the cochlea using PLGA nanoparticles. Laryngoscope 115: 2000-2005. [Crossref]

38. Fu QJ, Nogaki G (2005) Noise susceptibility of cochlear implant users: the role of spectral resolution and smearing. J Assoc Res Otolaryngol 6: 19-27. [Crossref]

39. Kallweit N, Baumhoff P, Krueger A, Tinne N, Kral A, et al. (2016) Optoacoustic effect is responsible for laser-induced cochlear responses. Sci Rep 6: 28141.

40. Hernandez VH, Gehrt A, Reuter K, Jing Z, Jeschke M, et al. (2014) Optogenetic stimulation of the auditory pathway. J Clin Invest 124: 1114-1129. [Crossref]

41. Roemer, A, Köhl, U, Majdani, O, Klöß, S, Falk, C, et al. (2016) Biohybrid cochlear implants in human neurosensory restoration. Stem Cell Res Ther 7: 148. [Crossref]

42. Gnecchi M, Zhang Z, Ni A, Dzau VJ (2008) Paracrine mechanisms in adult stem cell signaling and therapy. Circ Res 103: 1204-1219. [Crossref]

43. Martino G, Pluchino S, Bonfanti L, Schwartz M (2011) Brain regeneration in physiology and pathology: the immune signature driving therapeutic plasticity of neural stem cells. Physiol Rev 91: 1281-1304. [Crossref]

44. Schurzig D, Webster RJ 3rd, Dietrich MS, Labadie RF (2010) Force of cochlear implant electrode insertion performed by a robotic insertion tool: comparison of traditional versus Advance Off-Stylet techniques. Otol Neurotol 31: 1207-1210. [Crossref]

45. Schurzig D, Labadie RF, Hussong A, Rau TS, Webster RJ 3rd. (2012) Design of a tool integrating force sensing with automated insertion in cochlear implantation. IEEE ASME Trans Mechatron 17: 381-389. [Crossref]

46. Zhang J, Wei W, Ding J, Roland JT Jr, Manolidis S, et al. (2010) Inroads toward robotassisted cochlear implant surgery using steerable electrode arrays. Otol Neurotol 31 1199-1206. [Crossref]

Copyright: (C2019 Elgandy MS. This is an open-access article distributed under the terms of the Creative Commons Attribution License, which permits unrestricted use, distribution, and reproduction in any medium, provided the original author and source are credited. 